\title{
The impact of eliminating age inequalities in stage at diagnosis on breast cancer survival for older women
}

\author{
M J Rutherford ${ }^{\star, 1}$, G A Abel ${ }^{2}$, D C Greenberg ${ }^{2,3}$, P C Lambert ${ }^{1,4}$ and G Lyratzopoulos ${ }^{2,5}$ \\ ${ }^{1}$ Department of Health Sciences, University of Leicester, Leicester LE1 7RH UK; ${ }^{2}$ Cambridge Centre for Health Services Research, \\ Department of Public Health and Primary Care, University of Cambridge, Cambridge CB2 OSR, UK; ${ }^{3}$ National Cancer Registration \\ Service, Public Health England, Eastern Office, Cambridge CB22 3AD, UK; ${ }^{4}$ Department of Medical Epidemiology and \\ Biostatistics, Karolinska Institutet, Stocholm SE-171 77, Sweden and ${ }^{5}$ Health Behaviour Research Centre, Department of \\ Epidemiology and Public Health, University College London, UCL, 1-19 Torrington Place, London WC1E 6BT, UK
}

Background: Older women with breast cancer have poorer relative survival outcomes, but whether achieving earlier stage at diagnosis would translate to substantial reductions in mortality is uncertain.

Methods: We analysed data on East of England women with breast cancer (2006-2010) aged $70+$ years. We estimated survival for different stage-deprivation-age group strata using both the observed and a hypothetical stage distribution (assuming that all women aged $75+$ years acquired the stage distribution of those aged 70-74 years). We subsequently estimated deaths that could be postponed beyond 5 years from diagnosis if women aged $75+$ years had the hypothetical stage distribution. We projected findings to the English population using appropriate age and socioeconomic group weights.

Results: For a typically sized annual cohort in the East of England, 27 deaths in women with breast cancer aged $75+$ years can be postponed within 5 years from diagnosis if their stage distribution matched that of the women aged $70-74$ years (4.8\% of all 566 deaths within 5 years post diagnosis in this population). Under assumptions, we estimate that the respective number for England would be 280 deaths (5.0\% of all deaths within 5 years post diagnosis in this population).

Conclusions: The findings support ongoing development of targeted campaigns aimed at encouraging prompt presentation in older women.

Across Europe, older patients have benefited least from the substantial improvements in cancer survival in recent decades (Quaglia et al, 2009). Age gaps in cancer survival have been greatest in the UK and Ireland (De Angelis et al, 2014). Although some of these age inequalities may reflect sub-optimal management (Lavelle et al, 2007), another part may reflect that older patients are diagnosed at more advanced disease stage. For example, in England, older persons are at a greater risk of advanced stage at diagnosis of melanoma, endometrial and breast cancers (Lyratzopoulos et al, 2013a).
In England, about 6000 deaths in women aged 70 years or over are attributed to breast cancer each year (Office for National Statistics, 2013), and around a third of all new diagnoses of breast cancer occur in this age group (Cancer Research UK (CRUK) Website, 2014). In theory, decreasing the proportion of older women who are diagnosed with advanced-stage breast cancer should help prolong survival. Establishing the size of anticipated reduction in mortality using empirical evidence from population-based cohorts would be useful. Against this background, we have set out to examine the potential for preventing

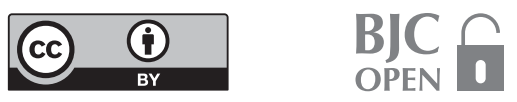


deaths within 5 years from diagnosis of breast cancer in older women that could result from eliminating age inequalities in stage at diagnosis.

\section{MATERIALS AND METHODS}

Data. We analysed time from diagnosis to death for East of England women aged 70 years or over with a new diagnosis of breast cancer (International Classification of Diseases-10 site code C50) during 2006-2010, with follow-up on mortality until 15 March 2012. As described previously (Rutherford et al, 2013b), the data were extracted from the (former) Eastern Cancer Registration and Information Centre (ECRIC), a cancer registry covering a population of $\sim 5.7$ million across the East of England region. Stage at diagnosis was assigned by medical practitioners with specialist expertise, based on clinical, imaging and pathological information according to the TNM classification (Sobin and Fleming, 1997). Socioeconomic status groups (one least deprived, five most deprived) were defined ecologically, using national quintiles of the income domain of the Index of Multiple Deprivation 2010 score of the Lower Super Output Area of patients' residence at diagnosis (Department for Communities and Local Government, 2011). We categorised age into four groups: $70-74,75-79,80-84$ and $85+$ years. Deprivation and age information were available for all patients. The data on age group and deprivation are tabulated in Table 1.

Analysis. We used a similar approach to Rutherford et al (2013b) but with matching of stage distributions across age rather than deprivation groups. Full details of the method are described in the methods section and appendix of the previously published paper (Rutherford et al, 2013b). Briefly, we fitted a flexible parametric excess mortality model (Nelson et al, 2007; Royston and Lambert, 2011) to estimate the effect of age, stage and deprivation status on excess mortality. We then calculated the proportion of women diagnosed at each stage for all deprivation-age group strata to obtain the relevant weights for the stage standardisation. Using the model estimates and these weights, we calculated stage-standardised survival for each age and deprivation group using two different stage distributions; one based on the observed stage distribution in each category and the second based on the stage distribution (in the respective deprivation group) for women aged 70-74 years. We calculated the difference between these two standardised survival estimates while accounting for other cause mortality, and we report the number of deaths postponed beyond given points during the follow-up period (i.e., beyond 5 years) by 'equalising' the stage distribution of each age group to match that of women in the 70- to 74-year group. We used a complete case analysis approach and deprivation quintile-specific life-tables for all survival estimates (Cancer Research UK Cancer Survival Group, 2006). Confidence intervals for the number of postponable deaths were calculated using the delta method using a similar approach to that described by Seppa et al (2012).

We also approximated the number of deaths that could be postponed in the whole of England rather than just the East of England region by appropriately weighting the estimates (Rutherford et al, 2013b) to match the age and socioeconomic group distribution for those aged $70+$ years in England as a whole. This involved re-weighting the estimates to account for sample size and compositional differences (e.g., regarding deprivation group) between the populations of East of England and England.

Supplementary analysis. We examined the potential for confounding by tumour type by exploring associations between age group and morphology, and morphology and stage. Using International Classification of Diseases-Oncology morphology
Table 1. Stage distributions across age and deprivation groups

\begin{tabular}{|c|c|c|c|c|c|}
\hline & Stage I & Stage II & Stage III & Stage IV & Total \\
\hline \multicolumn{6}{|c|}{ Most affluent } \\
\hline 70-74 & $182(41.27)$ & $200(45.4)$ & $40(9.1)$ & $19(4.3)$ & 441 (100) \\
\hline 75-79 & $173(37.0)$ & $220(47.0)$ & 55 (11.8) & $20(4.3)$ & $468(100)$ \\
\hline $80-84$ & $103(32.2)$ & 155 (48.4) & 38 (11.9) & $24(7.5)$ & 320 (100) \\
\hline $85+$ & $70(22.7)$ & $168(54.6)$ & $47(15.3)$ & $23(7.5)$ & $308(100)$ \\
\hline \multicolumn{6}{|c|}{ Deprivation group 2} \\
\hline 70-74 & $183(39.1)$ & $220(47.0)$ & $55(11.8)$ & $20(4.3)$ & $468(100)$ \\
\hline 75-79 & $145(30.8)$ & $133(44.9)$ & $31(10.5)$ & $21(7.1)$ & $471(100)$ \\
\hline $80-84$ & $93(26.7)$ & $163(46.8)$ & 50 (14.4) & 42 (12.1) & $348(100)$ \\
\hline $85+$ & $91(24.1)$ & $185(48.9)$ & $67(17.7)$ & $35(9.3)$ & $378(100)$ \\
\hline \multicolumn{6}{|c|}{ Deprivation group 3} \\
\hline $70-74$ & $174(39.0)$ & $193(43.3)$ & $38(8.5)$ & $41(9.2)$ & $446(100)$ \\
\hline 75-79 & $158(35.5)$ & $197(44.3)$ & $49(11.0)$ & $41(9.2)$ & 445 (100) \\
\hline 80-84 & $118(29.4)$ & $190(47.3)$ & 47 (11.7) & $47(11.7)$ & $402(100)$ \\
\hline $85+$ & $94(23.6)$ & $199(50.0)$ & $68(17.1)$ & 37 (9.3) & $398(100)$ \\
\hline \multicolumn{6}{|c|}{ Deprivation group 4} \\
\hline $70-74$ & $111(37.5)$ & $133(44.9)$ & $31(10.5)$ & $21(7.1)$ & $296(100)$ \\
\hline 75-79 & $91(27.7)$ & $156(47.6)$ & $36(11.0)$ & 45 (13.7) & $328(100)$ \\
\hline 80-84 & 69 (24.0) & 149 (51.9) & 41 (14.3) & $28(9.8)$ & $287(100)$ \\
\hline $85+$ & $71(22.9)$ & $158(51.0)$ & $63(20.3)$ & $18(5.8)$ & $310(100)$ \\
\hline \multicolumn{6}{|c|}{ Most deprived } \\
\hline 70-74 & $41(40.6)$ & $43(42.6)$ & $9(8.9)$ & $8(7.9)$ & $101(100)$ \\
\hline 75-79 & 32 (33.3) & 45 (46.9) & $12(12.5)$ & $7(7.29)$ & $96(100)$ \\
\hline 80-84 & 19 (25.3) & 37 (49.3) & $16(21.33)$ & $3(4.0)$ & 75 (100) \\
\hline $85+$ & $19(20.7)$ & 39 (42.4) & $26(28.3)$ & $8(8.7)$ & $92(100)$ \\
\hline \multicolumn{6}{|c|}{ Total (over deprivation groups) } \\
\hline $70-74$ & $691(39.4)$ & $769(43.9)$ & $172(9.8)$ & $120(6.9)$ & $1752(100)$ \\
\hline 75-79 & 599 (33.1) & 856 (47.4) & $203(11.2)$ & $150(8.3)$ & $1808(100)$ \\
\hline 80-84 & $402(28.1)$ & $694(48.5)$ & $192(13.4)$ & $144(10.1)$ & 1432 (100) \\
\hline $85+$ & $345(23.22)$ & $749(50.4)$ & $271(18.2)$ & $121(8.1)$ & $1486(100)$ \\
\hline \multicolumn{6}{|c|}{ Total (overall) } \\
\hline $70+$ & 2037 (31.4) & 3068 (47.4) & 838 (12.9) & $535(8.26)$ & $6478(100)$ \\
\hline
\end{tabular}

codes, four tumour groups (infiltrating ductal carcinoma, infiltrating lobular carcinoma, mixed infiltrating ductal and lobular carcinoma, and other unspecified types) were used for this analysis.

\section{RESULTS}

There were 6478 women aged $\geqslant 70$ years with complete information on stage at diagnosis included in subsequent analysis ( $88 \%$ of an initial total of 7331 women). The proportion of women with missing stage information was $6.2 \%, 7.6 \%, 12.8 \%$ and $20 \%$ for age groups 70-74 years, 75-79 years, 80-84 years and 85 + years, respectively. As previously reported (Lyratzopoulos et al, 2013a), among women with known stage, the proportion diagnosed in earlier stage progressively decreases with age over 70 years. For example, the proportion of women diagnosed at stage I is $39 \%$ for women aged $70-74$ years, whereas it is only $23 \%$ for the $85+$ age group; these age gradients are similar across deprivation groups (Table 1).

Stage-specific survival is markedly different for stage I compared with stages III or IV, across age groups (Figure 1). Therefore, if the distribution of stage at diagnosis for the older women matched that of those aged 70-74 years, there should be measurable improvements in survival, translating to a number of all-cause deaths that could be postponed.

Figure 1 shows the stage-specific survival for women aged 70 $74,75-79,80-84$ and $85+$ years. Relative survival for women diagnosed at stage I is nearly $100 \%$ during the entire follow-up period (i.e., up to 5 years from diagnosis) and for all age groups. 

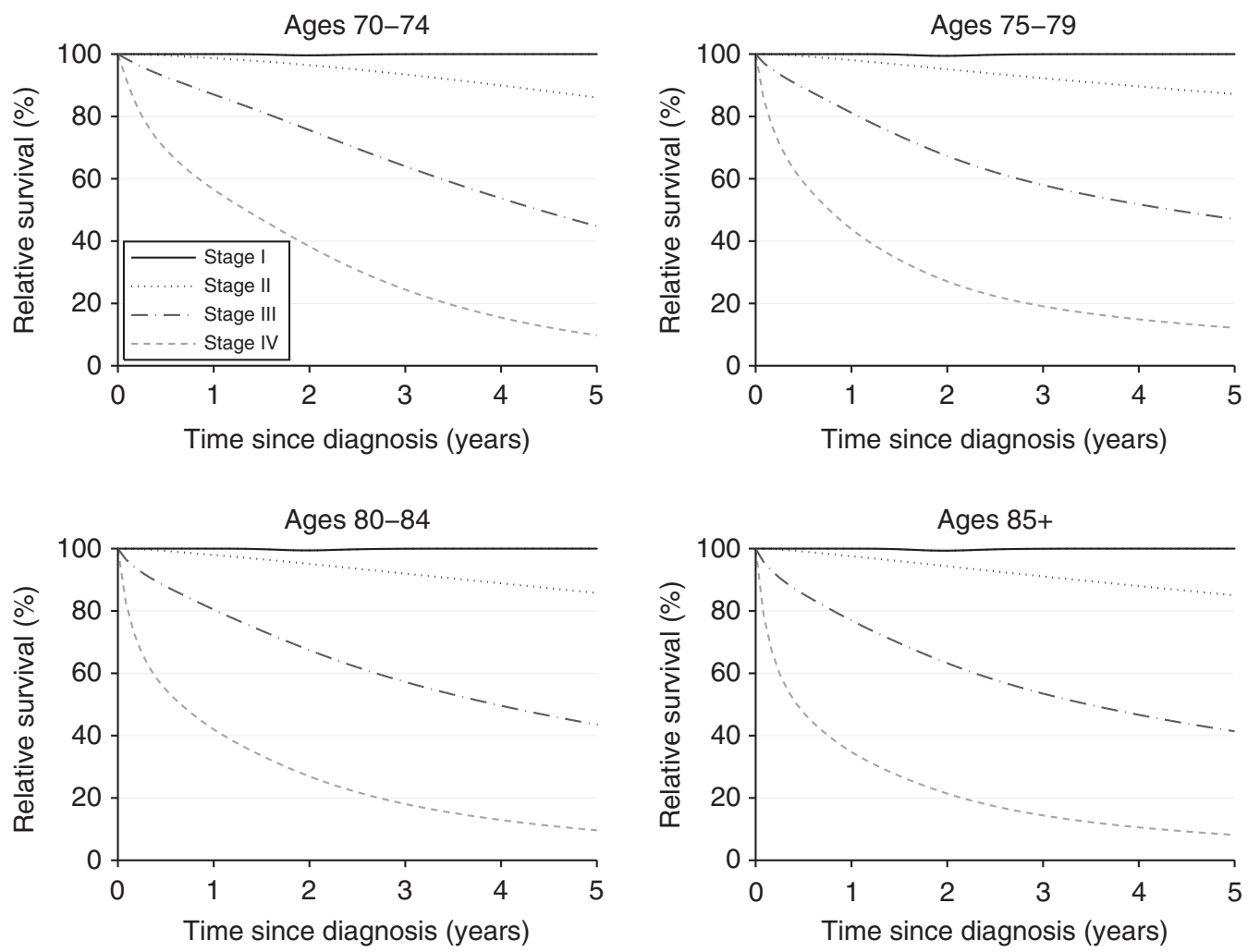

Figure 1. Stage-specific survival across the age groups 70-74, 75-79, 80-84 and $85+$ years.

In other words, women diagnosed at stage I experience almost no excess mortality compared with those in the general population without breast cancer. Stage-specific survival is fairly similar across all age groups (Figure 1). The relative survival for patients diagnosed at stage III or IV is quite poor across all age groups (for example, the 5-year relative survival for women diagnosed at stage III is $\sim 40 \%$, Figure 1 ), indicating that these women have a considerable excess mortality owing to breast cancer.

Figure 2 shows the number of deaths that can be postponed beyond certain points during the follow-up period by achieving earlier stage distribution in the three older age groups (i.e., by matching their stage distribution to that of women aged 70-74 years within the same deprivation groups). Figure 2A shows the relevant estimates for the East of England for (a typical annual cohort of 1296 cases) showing that 27 deaths (point estimate of 27.2 with 95\% CI 25.2 to 29.2) can be postponed to a time point beyond 5 years from diagnosis. This would represent $4.8 \%$ of all 566 deaths calculated to occur within 5 years from diagnosis in women with breast cancer aged $>70$ years in East of England. Figure 2B shows the re-weighted estimates for England using 2009 incidence figures for those aged $\geqslant 70$ years-about 280 deaths postponed beyond 5 years among older women, or $5.0 \%$ of all deaths in the respective population.

\section{DISCUSSION}

There is potential for reducing excess mortality from breast cancer by eliminating age inequalities in stage at diagnosis in women aged 70 years or over. This reduction in excess mortality will have a direct impact on all-cause survival estimates, and we estimate that around 280 deaths would be postponed beyond 5 years for a typical annual cohort size of older $(70+)$ women with breast cancer in England.

Strengths of our study include the use of a large populationbased sample with good quality and completeness of information
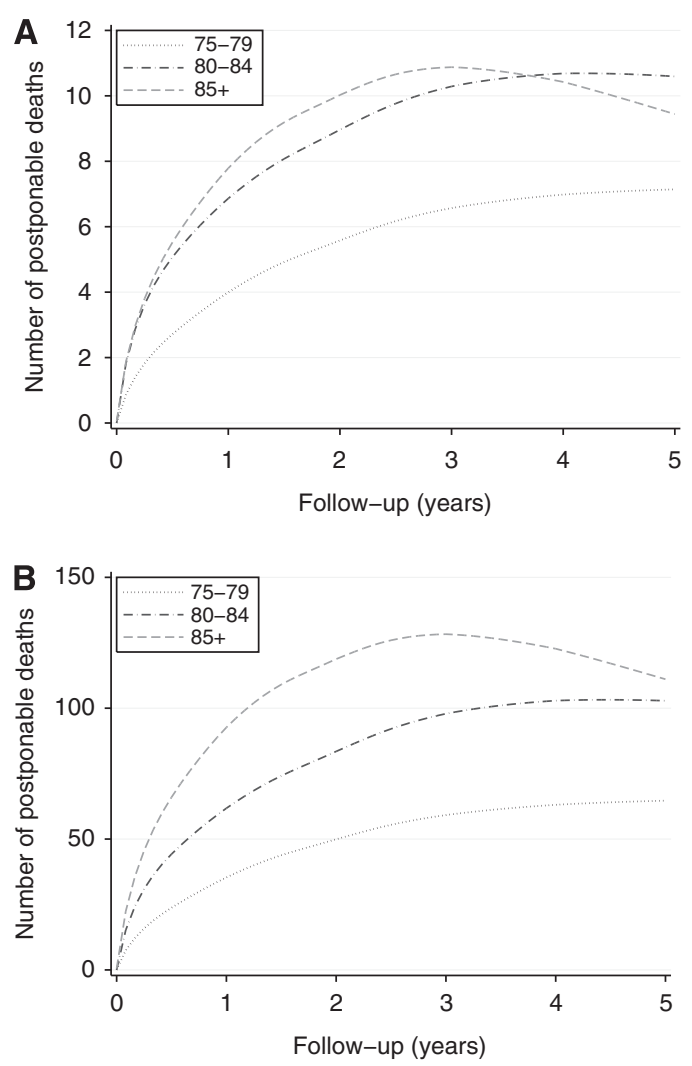

Figure 2. Number of avoidable deaths within 5 years from diagnosis of cancer. (A) East of England. (B) England. Note the different $y$-axis scales in the two sub-figures. Total values across all age groups at 5 years: 27.17 deaths in East of England, 278.59 deaths for England. 
on stage at diagnosis, and the use of a flexible parametric model, allowing for the smooth estimation of excess mortality throughout the follow-up period while appropriately accounting for the effects of deprivation and age (Rutherford et al, 2013a). The methodology that we have used in this paper can help support monitoring of the impact of population-based early awareness and detection interventions (Be Clear On Cancer-Breast Cancer in Over 70s Campaign, 2014).

We restricted our study population to a historical cohort of East of England women (2006-10) with both high level of completeness of information on stage at diagnosis and an adequate follow-up period. As recording of stage information becomes increasingly complete and consistent across England, future work should aim to encompass more recent cohorts of patients using nationwide data. Limiting to regional data means that we have small numbers for some of the cells in Table 1, particularly for the most-deprived patients and late-stage disease. Extrapolating the regional estimates to England as a whole makes the assumption that patients with breast cancer in England do not differ from those in the East of England in terms of expected survival, the effect of the covariates on relative survival and the distribution of stage at diagnosis. Overall, these assumptions appear to be fairly reasonable, particularly given only modest variation in short-term relative survival for breast cancer patients between the English regions (Rachet et al, 2009).

To further aid interpretation and to contextualise the findings, we would like to draw attention to the fact that all deaths estimated in our paper are actually postponed (an alternative term used for this measure is avoidable deaths) - the entire cohort will eventually diminish to 0 if follow-up is extended long enough. For women aged $85+$ years in particular, the estimated number of postponed deaths begins to decrease within 5 years from diagnosis (Figure 2), as other cause mortality is particularly high in that age group. Further, the findings need to be interpreted in the context of previous work examining potential survival gains by eliminating socioeconomic inequalities in stage at diagnosis of breast cancer among women of any age (Rutherford et al, 2013b). In contrast, the present study solely focuses on age inequalities in stage at diagnosis among older women. Combined elimination of inequalities in stage at diagnosis both by socioeconomic and older age groups would be associated with a greater number of deaths that can be postponed beyond 5 years from diagnosis in the population.

Three issues deserve further discussion. First, our method does not make any adjustment for differences in tumour type across age groups. We have shown in supplementary analyses that differences in morphology by age are unlikely to be large (Supplementary Online Material Tables 1 and 2). Although there are some differences in the stage distribution across morphology groups (Supplementary Online Material Table 3), tumour type does not have a large impact on survival in addition to stage. Although breast cancer is known to be a heterogeneous disease, most of the tumour type differences relate to pre-menopausal compared with postmenopausal women (Anderson et al, 2014). In contrast, our study population only included (post-menopausal) women aged $\geqslant 70$ years.

Second, we excluded women below the age of 70 years owing to the impact of screening. Screen detection has an impact on stage at diagnosis (the great majority of screen-detected cases will be diagnosed at stages I or II) and could introduce differential lead time bias in the survival estimates. Evidence does not, at least currently, support the offer of screening in older women (Independent UK Panel on Breast Cancer Screening, 2012; de Glas et al, 2014), although this is the subject of ongoing research (CRUK Website-A study to evaluate an age extension to the NHS Breast Screening Programme, 2014). However, our method could be sensitive to the use of screening in our sample. The proportion of women in our study population who were screening-detected differs by age $(26 \%, 12 \%, 3 \%$ and $1 \%$ for women aged $70-74,75-$ $79,80-84$ and $85+$ years, respectively). It is likely that these differences largely reflect age variation in use (as opposed to effectiveness) of screening. Owing to the level of screening use in women aged 70-74, our global estimate of avoidable all-cause deaths in women aged 70 years or older may be upwardly inflated. Nevertheless, further potential gains in the number of postponable deaths in women older than 70 could be made by achieving a (more favourable) stage distribution that would be seen for patients aged 65-69 years. However, this comparison is made difficult by the high use of screening in this group (over half of all women aged 65-69 are detected by screening).

Finally, older age is associated with both a greater risk of advanced stage at diagnosis and missing stage information. Therefore, and as our findings are based on a complete case analysis, the true potential for avoidable deaths may have been under-estimated. In addition, the higher concentration of (excluded) cases with missing stage in older age groups leads to differential reduction of the sample size, again resulting in underestimation of the true potential for reducing deaths in older women. On the other hand, women with missing stage disease also have fairly poor survival, and therefore our stage-specific survival estimates may be upwardly biased to a small degree.

In their largest part, age differentials in stage at diagnosis of breast cancer in women aged $\geqslant 70$ years are likely to reflect age differences in promptness of presentation (i.e., age differences in the patient interval) as opposed to differences in diagnostic intervals after presentation. This is because after seeking medical help with relevant symptoms, the great majority of English women subsequently diagnosed with breast cancer are referred very promptly independently of their age (median primary care interval $=0$ days, interquartile range: $0-1$; Lyratzopoulos et al, 2013b), with concordant evidence from Scotland (Baughan et al, 2009). Further, older age confers no disadvantage in speed of specialist referral (Lyratzopoulos et al, 2012).

We did not have information on co-morbidity in our study, which increases with age. However, as we estimate survival within age groups, this will lessen the impact of differential co-morbidity by age. Further, evidence from the US indicates that greater levels of co-morbidity may be associated with earlier stage at diagnosis of breast and colorectal cancer, possibly because of greater opportunities to detect cancer early in more co-morbid patients through more frequent care appointments (Keating et al, 2005, Zafar et al, 2008). Therefore, the fact that older women could be expected to have greater levels of co-morbidity could facilitate rather than impede earlier stage at diagnosis.

Our findings therefore provide support both for further research to establish the causes of age inequalities in stage at diagnosis of breast cancer and ongoing public health campaigns aimed to encourage prompt presentation in older women (Be Clear On Cancer-Breast Cancer in Over 70s Campaign, 2014). Such campaigns can, for example, aim to increase awareness of cancer signs and symptoms (a conceptual surrogate of the patient interval; Lyratzopoulos, 2014), which is lower among older patients (Robb et al, 2009; Forbes et al, 2011), or they can aim to increase the public understanding of the age-dependent nature of breast cancer risk, which is low in the general English population (Forbes et al, 2013). Subject to appropriate development, validation and evaluation, campaigns that target women aged $70+$ years should be encouraged, as they can lead to substantial reductions in avoidable mortality through earlier stage diagnosis of breast cancer in these women.

\section{ACKNOWLEDGEMENTS}

This article is an independent research supported by different funding bodies, beyond the authors' own employing organisations. MJR was partially funded by a Cancer Research UK Postdoctoral 
Fellowship (CRUK_A13275). GL is supported by a Postdoctoral Fellowship award by the National Institute for Health Research (NIHR PDF-2011-04-047) to end of 2014 and a Cancer Research UK Clinician Scientist Fellowship award (A18180) from 2015. We thank all staff at the National Cancer Registration Service, Public Health England, Eastern Office who helped collect and code data used in this study. We particularly acknowledge the help of Dr Clement H Brown and Dr Brian A Rous who were responsible for staging.

\section{CONFLICT OF INTEREST}

The authors declare no conflict of interest.

\section{DISCLAIMER}

The views expressed in this publication are those of the authors and not necessarily those of the NHS, the National Institute for Health Research, the Department of Health, Cancer Research UK, or any other organisation.

\section{REFERENCES}

Anderson WF, Rosenberg PS, Prat A, Perou CM, Sherman ME (2014) How many etiological subtypes of breast cancer: two, three, four, or more? J Natl Cancer Inst 106(8) dju165 doi: 10.1093/jnci/dju165.

Baughan P, O'Neill B, Fletcher E (2009) Auditing the diagnosis of cancer in primary care: the experience in Scotland. Br J Cancer 101(Suppl 2): S87-S91.

Be Clear On Cancer-Breast Cancer in Over 70s Campaign (2014) 'http://www.nhs.uk/be-clear-on-cancer/breast-cancer/home'.

Cancer Research UK Cancer Survival Group (2006) 'Life tables for cancer survival analysis'London School of Hygiene \& Tropical Medicine: London, UK.

CRUK Website: http://www.cancerresearchuk.org/cancer-info/cancerstats/ types/breast/incidence/uk-breast-cancer-incidence-statistics\#age (accessed 9 November 2014).

CRUK Website-A study to evaluate an age extension to the NHS Breast Screening Programme (2014) http://www.cancerresearchuk.org/ about-cancer/trials/a-study-to-evaluate-an-age-extension-of-the-nhsbreast-screening-programme.

De Angelis R, Sant M, Coleman MP, Francisci S, Baili P, Pierannunzio D, Trama A, Visser O, Brenner H, Ardanaz E, Bielska-Lasota M, Engholm G, Nennecke A, Siesling S, Berrino F, Capocaccia R. EUROCARE-5 Working Group (2014) Cancer survival in europe 1999-2007 by country and age: results of EUROCARE-5-a population-based study. Lancet Oncol 15(1): 23-34.

de Glas NA, de Craen AJM, Bastiaannet E, Op 't Land EG, Kiderlen M, van de Water W, Siesling S, Portielje JEA, Schuttevaer HM, de Bock GTH, van de Velde CJH, Liefers G-J (2014) Effect of implementation of the mass breast cancer screening programme in older women in the Netherlands: population based study. BMJ 349: g5410.

Department for Communities and Local Government (2011) 'English indices of deprivation 2010'.

Forbes LJL, Linsell L, Atkins L, Burgess C, Tucker L, Omar L, Ramirez AJ (2011) A promoting early presentation intervention increases breast cancer awareness in older women after 2 years: a randomised controlled trial. Br J Cancer 105(1): 18-21.

Forbes LJL, Simon AE, Warburton F, Boniface D, Brain KE, Dessaix A, Donnelly C, Haynes K, Hvidberg L, Lagerlund M, Lockwood G, Tishelman C, Vedsted P, Vigmostad MN, Ramirez AJ, Wardle J. ICBP Module 2 Working Group (2013) Differences in cancer awareness and beliefs between Australia, Canada, Denmark, Norway, Sweden and the UK (the international cancer benchmarking partnership): do they contribute to differences in cancer survival? Br J Cancer 108(2): 292-300.
Independent UK Panel on Breast Cancer Screening (2012) The benefits and harms of breast cancer screening: an independent review. Lancet 380(9855): 1778-1786.

Keating NL, Landrum MB, Ayanian JZ, Winer EP, Guadagnoli E (2005) The association of ambulatory care with breast cancer stage at diagnosis among Medicare beneficiaries. J Gen Intern Med 20(1): 38-44.

Lavelle K, Todd C, Moran A, Howell A, Bundred N, Campbell M (2007) 'Non-standard management of breast cancer increases with age in the UK: a population based cohort of women $>$ or $=65$ years'. $B r$ J Cancer $96(8)$ : $1197-1203$

Lyratzopoulos G (2014) 'Markers and measures of timeliness of cancer diagnosis after symptom onset: a conceptual framework and its implications.'. Cancer Epidemiol 38(3): 211-213.

Lyratzopoulos G, Abel GA, Brown C, Rous B, Vernon S, Roland M, Greenberg D (2013a) Socio-demographic inequalities in stage of cancer diagnosis: evidence from patients with female breast, lung, colon, rectal, prostate, renal, bladder, melanoma, ovarian and endometrial cancer. Ann Oncol 24(3): 843-850.

Lyratzopoulos G, Abel GA, McPhail S, Neal RD, Rubin GP (2013b) Measures of promptness of cancer diagnosis in primary care: secondary analysis of national audit data on patients with 18 common and rarer cancers. Br J Cancer 108(3): 686-690.

Lyratzopoulos G, Neal RD, Barbiere JM, Rubin GP, Abel GA (2012) Variation in number of general practitioner consultations before hospital referral for cancer: findings from the 2010 National Cancer Patient Experience Survey in England. Lancet Oncol 13(4): 353-365.

Nelson CP, Lambert PC, Squire IB, Jones DR (2007) Flexible parametric models for relative survival, with application in coronary heart disease. Stat Med 26(30): 5486-5498.

Office for National Statistics (2013) 'Mortality statistics: Deaths registered in England and Wales (series dr), 2012. http://www.ons.gov.uk/ons/ publications/re-reference-tables.html?edition=tcm\%3a77-325289.

Quaglia A, Tavilla A, Shack L, Brenner H, Janssen-Heijnen M, Allemani C, Colonna M, Grande E, Grosclaude P, Vercelli M. EUROCARE Working Group (2009) 'The cancer survival gap between elderly and middle-aged patients in Europe is widening'. Eur J Cancer 45(6): 1006-1016.

Rachet B, Maringe C, Nur U, Quaresma M, Shah A, Woods LM, Ellis L, Walters S, Forman D, Steward J, Coleman MP (2009) Population-based cancer survival trends in england and wales up to 2007: an assessment of the NHS cancer plan for England. Lancet Oncol 10(4): 351-369.

Robb K, Stubbings S, Ramirez A, Macleod U, Austoker J, Waller J, Hiom S, Wardle J (2009) Public awareness of cancer in Britain: a population-based survey of adults. Br J Cancer 101(S2): S18-S23.

Royston P, Lambert PC (2011) Flexible Parametric Survival Analysis Using Stata: Beyond the Cox Model. Stata Press. http://www.stata.com/bookstore/ flexible-parametric-survival-analysis-stata/.

Rutherford MJ, Crowther MJ, Lambert PC (2013a) The use of restricted cubic splines to approximate complex hazard functions in the analysis of time-to-event data: a simulation study. J Stat Comput Sim 85: 777-793.

Rutherford MJ, Hinchliffe SR, Abel GA, Lyratzopoulos G, Lambert PC, Greenberg DC (2013b) 'How much of the deprivation gap in cancer survival can be explained by variation in stage at diagnosis: an example from breast cancer in the East of England'. Int J Cancer 133(9): 2192-2200.

Seppa K, Hakulinen T, Laara E (2012) 'Avoidable deaths and random variation in patients' survival'. Br J Cancer 106(11): 1846-1849.

Sobin LH, Fleming ID (1997) 'TNM classification of malignant tumors, fifth edition (1997)'. Cancer 80(9): 1803-1804.

Zafar SY, Abernethy AP, Abbott DH, Grambow SC, Marcello JE, Herndon 2nd JE, Rowe KL, Kolimaga JT, Zullig LL, Patwardhan MB, Provenzale DT (2008) Comorbidity, age, race and stage at diagnosis in colorectal cancer: a retrospective, parallel analysis of two health systems. BMC Cancer 8: 345 .

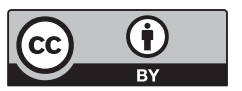

This work is licensed under the Creative Commons Attribution 4.0 International License. To view a copy of this license, visit http://creativecommons.org/licenses/by/4.0/

Supplementary Information accompanies this paper on British Journal of Cancer website (http://www.nature.com/bjc) 